This is page 1

Printer: Opaque this

\title{
Geometric algebra and particle dynamics
}

\author{
José B. Almeida
}

\begin{abstract}
In a recent publication [1] it was shown how the geometric algebra $G_{4,1}$, the algebra of 5-dimensional space-time, can generate relativistic dynamics from the simple principle that only null geodesics should be allowed. The same paper showed also that Dirac equation could be derived from the condition that a function should be monogenic in that algebra; this construction of the Dirac equation allows a choice for the imaginary unit and it was suggested that different imaginary units could be assigned to the various elementary particles. An earlier paper 2] had already shown the presence of standard model gauge group symmetry in complexified $G_{1,3}$, an algebra isomorphic to $G_{4,1}$.

In this presentation I explore the possible choices for the imaginary unit in the Dirac equation to show that $S U(3)$ and $S U(2)$ symmetries arise naturally from such choices. The quantum numbers derived from the imaginary unit are unusual but a simple conversion allows the derivation of electric charge and isospin, quantum numbers for two families of particles. This association to elementary particles is not final because further understanding of the role played by the imaginary unit is needed.
\end{abstract}

Keywords: Dirac equation, monogenic functions, gauge group.

\section{Introduction}

Extracting the laws of physics from a small set of geometric postulates is an exciting activity which the author has been exercising for some time with great enthusiasm and led recently to the presentation of some interesting results 3 , 1]. In both of these papers the emphasis is on the dynamics of bodies formulated in $4 \mathrm{D}$ Euclidean space and its relation to general relativity (GR). It was shown how and when GR and 4D Euclidean metrics can be converted into each other and an equation was proposed to link the Euclidean metric to its sources. The 4D Euclidean formulation of dynamics is similar to Fermat's principle applied in 4 dimensions, which motivated the designation 4-dimensional optics (4DO) to this approach. The point of departure for the cited work is the 4-dimensional space constructed with the imposition of null displacement in 5D space with signature $(-++++)$.

AMS Subject Classification: 51P05; 81R50; 81Q05. 
Defining 4D space in this way leaves its signature undefined until one of the coordinates is expressed in terms of the other 4, allowing for both Minkowski and Euclidean signatures and providing the means for conversion between signatures. The second paper cited above examined monogenic functions [4] in $G_{4,1}$ algebra $^{1}$ to show that these verify Dirac's equation for a free particle. Similarly, if one turns his attention to Euclidean 4-space, the same solutions become plane waves that extend 4DO concept into wave optics.

It is generally accepted that any physics theory is based on a set of principles from which predictions are derived using established mathematical rules; the validity of such theory depends on agreement between predictions and observed physical reality. In that sense this paper, as well as those cited above, does not formulate physical theories because it does not presume any physical principles. This paper discusses geometry; all along the paper, in several occasions, a parallel is made with the physical world by assigning a physical meaning to geometric entities and this allows predictions to be made. However the validity of derivations and overall consistency of the exposition is independent of prediction correctness. The only postulates that are made are of a geometrical nature and can be summarized in the definition of the space we are going to work with; this is the 5 -dimensional space with signature $(-++++)$, where we investigate monogenic functions. Monogenic functions provide a more fundamental constraint than null subspace and the latter condition can be shown to result from the former under certain conditions. The choice of this geometric space does not imply any assumption for physical space up to the point where geometric entities like coordinates and geodesics start being assigned to physical quantities like distances and trajectories. Some of those assignments are carried over from previous work; for instance we have already established that coordinate $x^{0}$ is to be taken as time and coordinate $x^{4}$ as proper time [1].

Mapping between geometry and physics is facilitated if one chooses to work always with non-dimensional quantities; this is done with a suitable choice for standards for the fundamental units. From this point onwards all problems of dimensional homogeneity are avoided through the use of normalizing factors listed below for all units, defined with recourse to the fundamental constants: $\hbar \rightarrow$ Planck constant divided by $2 \pi, G \rightarrow$ gravitational constant, $c \rightarrow$ speed of light and $e \rightarrow$ proton charge.

\begin{tabular}{c|c|c|c} 
Length & Time & Mass & Charge \\
\hline$\sqrt{\frac{G \hbar}{c^{3}}}$ & $\sqrt{\frac{G \hbar}{c^{5}}}$ & $\sqrt{\frac{\hbar c}{G}}$ & $e$
\end{tabular}

\footnotetext{
${ }^{1} \mathrm{I}$ use the convention that first/second subindex indicates the number of positive/negative norm frame vectors. This convention was adopted in my previous papers, before I was aware that some people followed the opposite one.
} 
This normalization defines a system of non-dimensional units (Planck units) with important consequences, namely: 1) All the fundamental constants, $\hbar, G, c, e$, become unity; 2) a particle's Compton frequency, defined by $\nu=m c^{2} / \hbar$, becomes equal to the particle's mass; 3$)$ the frequent term $G M /\left(c^{2} r\right)$ is simplified to $M / r$.

The consideration of monogenic functions in the 5-dimensional space defined above will be seen to provide several bridges to the physical world, namely in the areas of particle electrodynamics and the standard model. The exposition makes full use of an extraordinary and little known mathematical tool called geometric algebra (GA), a.k.a. Clifford algebra, which received an important thrust with the addition of calculus by David Hestenes [5]. A good introduction to GA can be found in Gull et al. [6] and the following paragraphs use basically the notation and conventions therein with adaptations to 5D hyperbolic space. A complete course on physical applications of GA can be downloaded from the internet 7]; the same authors published a more comprehensive version in book form [4].

\section{Introduction to geometric algebra}

Before we begin our exposition a difficult notation issue must be resolved. We are dealing with 5-dimensional space but we are simultaneously interested in two of its 4-dimensional and one 3-dimensional subspaces; ideally our choice of indices should clearly identify their ranges in order to avoid the need to specify them in every equation. The following diagram shows the index naming convention used in this paper.

$$
\frac{\imath}{\overbrace{0, \underbrace{\frac{1,2,3}{m}}_{i}, 4}^{\imath}}
$$

Indices in the range $\{0,4\}$ will be denoted with Greek letters $\iota, \kappa, \lambda$. Indices in the range $\{0,3\}$ will also receive Greek letters but chosen from $\mu, \nu, \xi$. For indices in the range $\{1,4\}$ we will use Latin letters $i, j, k$ and finally for indices in the range $\{1,3\}$ we will use also Latin letters chosen from $m, n, o$.

Einstein's summation convention will be adopted as well as the compact notation for partial derivatives $\partial_{\iota}=\partial / \partial x^{\iota}$. When convenient we will also make the assignments justified in Almeida [1]: $x^{0} \equiv t$ and $x^{4} \equiv \tau$. The geometric algebra $G_{4,1}$ of the hyperbolic 5-dimensional space we want to consider is generated by the frame of orthonormal vectors $\sigma_{\iota}$ verifying the 
relations

$$
\begin{aligned}
& \left(\sigma_{0}\right)^{2}=-1, \\
& \sigma_{0} \sigma_{i}+\sigma_{i} \sigma_{0}=0, \\
& \sigma_{i} \sigma_{j}+\sigma_{j} \sigma_{i}=2 \delta_{i j} .
\end{aligned}
$$

We will simplify the notation for basis vector products using multiple indices, i.e. $\sigma_{\iota} \sigma_{\kappa} \equiv \sigma_{\iota \kappa}$. The algebra is 32 -dimensional and is spanned by the basis

- 1 scalar, 1

- 5 vectors, $\sigma_{\iota}$

- 10 bivectors (area), $\sigma_{\iota \kappa}$

- 10 trivectors (volume), $\sigma_{\iota \kappa \lambda}$

- 5 tetravectors (4-volume), i $\sigma_{\iota}$

- 1 pseudoscalar (5-volume), $\mathrm{i} \equiv \sigma_{01234}$

Several elements of this basis square to unity:

$$
\left(\sigma_{i}\right)^{2}=\left(\sigma_{0 i}\right)^{2}=\left(\sigma_{0 i j}\right)^{2}=\left(\mathrm{i} \sigma_{0}\right)^{2}=1 ;
$$

and the remaining square to -1 :

$$
\left(\sigma_{0}\right)^{2}=\left(\sigma_{i j}\right)^{2}=\left(\sigma_{i j k}\right)^{2}=\left(\mathrm{i} \sigma_{i}\right)^{2}=\mathrm{i}^{2}=-1 .
$$

Note that the pseudoscalar i commutes with all the other basis elements while being a square root of -1 and plays the role of the scalar imaginary in complex algebra. When writing matrix expressions equivalent to geometric algebra we will represent the complex imaginary by the symbol $\mathrm{j}$, following the use of electrical engineering.

The geometric product of any two vectors $a=a^{\iota} \sigma_{\iota}$ and $b=b^{\kappa} \sigma_{\kappa}$ is evaluated making use of the distributive property

$$
a b=\left(-a^{0} b^{0}+\sum_{i} a^{i} b^{i}\right)+\sum_{\iota \neq \kappa} a^{\iota} b^{\kappa} \sigma_{\iota \kappa} ;
$$

and we notice it can be decomposed into a symmetric part, a scalar called the inner or interior product, and an anti-symmetric part, a bivector called the outer or exterior product.

$$
a b=a \cdot b+a \wedge b, \quad b a=a \cdot b-a \wedge b .
$$

Reversing the definition one can write inner and outer products as

$$
a \cdot b=\frac{1}{2}(a b+b a), \quad a \wedge b=\frac{1}{2}(a b-b a) .
$$


When a vector is operated with a multivector the inner product reduces the grade of each element by one unit and the outer product increases the grade by one. By convention the inner product of a vector and a scalar produces a vector.

The geometric algebra $G_{4,1}$ is isomorphic to the complex algebra of $4 \times 4$ matrices, designated by $M(4, C)$. When necessary we will invoke this isomorphism to simplify the exposition of some concepts, namely those related to symmetry. The following mapping between basis vectors and matrices will be used

$$
\begin{gathered}
\sigma_{0} \equiv\left(\begin{array}{cccc}
-\mathrm{j} & 0 & 0 & 0 \\
0 & \mathrm{j} & 0 & 0 \\
0 & 0 & -\mathrm{j} & 0 \\
0 & 0 & 0 & \mathrm{j}
\end{array}\right), \sigma_{1} \equiv\left(\begin{array}{cccc}
0 & 0 & 0 & 1 \\
0 & 0 & -1 & 0 \\
0 & -1 & 0 & 0 \\
1 & 0 & 0 & 0
\end{array}\right), \sigma_{2} \equiv\left(\begin{array}{cccc}
0 & \mathrm{j} & 0 & 0 \\
-\mathrm{j} & 0 & 0 & 0 \\
0 & 0 & 0 & -\mathrm{j} \\
0 & 0 & \mathrm{j} & 0
\end{array}\right) \\
\sigma_{3} \equiv\left(\begin{array}{cccc}
0 & 1 & 0 & 0 \\
1 & 0 & 0 & 0 \\
0 & 0 & 0 & 1 \\
0 & 0 & 1 & 0
\end{array}\right), \sigma_{4} \equiv\left(\begin{array}{cccc}
0 & 0 & 0 & -\mathrm{j} \\
0 & 0 & \mathrm{j} & 0 \\
0 & -\mathrm{j} & 0 & 0 \\
\mathrm{j} & 0 & 0 & 0
\end{array}\right) .
\end{gathered}
$$

We will encounter exponentials with multivector exponents; two particular cases of exponentiation are specially important. If $u$ is such that $u^{2}=-1$ and $\theta$ is a scalar

$$
\begin{aligned}
\mathrm{e}^{u \theta}= & 1+u \theta-\frac{\theta^{2}}{2 !}-u \frac{\theta^{3}}{3 !}+\frac{\theta^{4}}{4 !}+\ldots \\
= & 1-\frac{\theta^{2}}{2 !}+\frac{\theta^{4}}{4 !}-\ldots\{=\cos \theta\} \\
& +u \theta-u \frac{\theta^{3}}{3 !}+\ldots\{=u \sin \theta\} \\
= & \cos \theta+u \sin \theta .
\end{aligned}
$$

Conversely if $h$ is such that $h^{2}=1$

$$
\begin{aligned}
\mathrm{e}^{h \theta}= & 1+h \theta+\frac{\theta^{2}}{2 !}+h \frac{\theta^{3}}{3 !}+\frac{\theta^{4}}{4 !}+\ldots \\
= & 1+\frac{\theta^{2}}{2 !}+\frac{\theta^{4}}{4 !}+\ldots\{=\cosh \theta\} \\
& +h \theta+h \frac{\theta^{3}}{3 !}+\ldots\{=h \sinh \theta\} \\
= & \cosh \theta+h \sinh \theta .
\end{aligned}
$$

The exponential of bivectors is useful for defining rotations; a rotation of vector $a$ by angle $\theta$ on the $\sigma_{12}$ plane is performed by

$$
a^{\prime}=\mathrm{e}^{\sigma_{21} \theta / 2} a \mathrm{e}^{\sigma_{12} \theta / 2}=\tilde{R} a R
$$


the tilde denotes reversion and reverses the order of all products. As a check we make $a=\sigma_{1}$

$$
\begin{aligned}
\mathrm{e}^{-\sigma_{12} \theta / 2} \sigma_{1} \mathrm{e}^{\sigma_{12} \theta / 2} & =\left(\cos \frac{\theta}{2}-\sigma_{12} \sin \frac{\theta}{2}\right) \sigma_{1}\left(\cos \frac{\theta}{2}+\sigma_{12} \sin \frac{\theta}{2}\right) \\
& =\cos \theta \sigma_{1}+\sin \theta \sigma_{2} .
\end{aligned}
$$

Similarly, if we had made $a=\sigma_{2}$, the result would have been $-\sin \theta \sigma_{1}+$ $\cos \theta \sigma_{2}$.

If we use $B$ to represent a bivector belonging to Euclidean space and define its norm by $|B|=(B \tilde{B})^{1 / 2}$, a general rotation in 4-space is represented by the rotor

$$
R \equiv e^{-B / 2}=\cos \left(\frac{|B|}{2}\right)-\frac{B}{|B|} \sin \left(\frac{|B|}{2}\right) .
$$

The rotation angle is $|B|$ and the rotation plane is defined by $B$. A rotor is defined as a unitary even multivector (a multivector with even grade components only) which squares to unity; we are particularly interested in rotors with bivector components. It is more general to define a rotation by a plane (bivector) then by an axis (vector) because the latter only works in $3 \mathrm{D}$ while the former is applicable in any dimension.

In a general situation the frame vectors need not be orthonormed; we designate such frame by refractive index frame [1] and denote its vectors by the symbol $g_{\iota}$

$$
g_{\iota}=n_{\iota}^{\kappa} \sigma_{\kappa},
$$

where $n_{L}^{\kappa}$ is called the refractive index tensor. Refractive index frames are used for gravitational dynamics, which lies outside the scope of the present paper. In association with the refractive index frame we will now introduce the concept of reciprocal frame, with frame vectors $g^{\iota}$, which will be needed in several occasions along the paper. For our 5 -dimensional space the reciprocal frame is defined by the relations

$$
g^{\iota} \cdot g_{\kappa}=\delta^{\iota}{ }_{\kappa} .
$$

Following the procedure outlined in [4] to determine the reciprocal frame vectors, we define the 5 -volume pseudoscalar

$$
V=\bigwedge_{\iota=0}^{4} g_{\iota}=|V| \mathrm{i},
$$

where the big wedge symbol is used to make the exterior product of the $g_{\iota}$. The reciprocal frame vectors can then be found using the formula [4]

$$
g^{\iota}=\frac{(-1)^{(\iota+1)}}{|V|} \bigwedge_{\iota \neq \kappa} g_{\kappa} \mathrm{i} .
$$


Applying the definition to the $\sigma_{\iota}$ frame we are now working with, it is easy to see that the reciprocal frame is $\sigma^{0}=-\sigma_{0}, \sigma^{i}=\sigma_{i}$.

The first use we will make of the reciprocal frame is for the definition of a vector derivative. In any space

$$
\mathrm{D}=g^{\iota} \partial_{\iota}
$$

is a vector and as such can be left or right multiplied with other vectors or multivectors. For instance, when multiplied by vector $a$ on the right the result comprises scalar and bivector terms $\mathrm{D} a=\mathrm{D} \cdot a+\mathrm{D} \wedge a$. The scalar part can be immediately associated with the divergence and the bivector part is called the exterior derivative; in the particular case of Euclidean 3dimensional space it is possible to define the curl of a vector by $\operatorname{curl}(a)=$ $-\sigma_{123} \mathrm{D} \wedge$.

It will be convenient, sometimes, to use vector derivatives in subspaces of $5 \mathrm{D}$ space; these will be denoted by an upper index before the $\mathrm{D}$ and the particular index used determines the subspace to which the derivative applies; For instance ${ }^{m} \mathrm{D}=\sigma^{m} \partial_{m}=\sigma^{1} \partial_{1}+\sigma^{2} \partial_{2}+\sigma^{3} \partial_{3}$. In 5-dimensional space it will be useful to split the vector derivative into its time and 4dimensional parts

$$
\mathrm{D}=-\sigma_{0} \partial_{t}+\sigma^{i} \partial_{i}=-\sigma_{0} \partial_{t}+{ }^{i} \mathrm{D} .
$$

We define also second order differential operators, generally designated Laplacian, resulting from the product of the vector derivative by itself. The square of a vector is always a scalar and the vector derivative is no exception, so the Laplacian is a scalar operator, which consequently acts separately in each component of a multivector. For $4+1$ space it is

$$
\mathrm{D}^{2}=-\frac{\partial^{2}}{\partial t^{2}}+{ }^{i} \mathrm{D}^{2}
$$

One sees immediately that a 4-dimensional wave equation is obtained zeroing the Laplacian of some multivector function

$$
\mathrm{D}^{2} \psi=\left(-\frac{\partial^{2}}{\partial t^{2}}+{ }^{i} \mathrm{D}^{2}\right) \psi=0
$$

We will be returning to this wave equation but for the moment we must spend some time examining the symmetries of $G_{4,1}$.

\section{Symmetries of $G_{4,1}$ algebra}

In this algebra it is possible to find a maximum of four mutually annihilating idempotents, which generate with 0 an additive group of order 16; for a demonstration see Lounesto [8, section 17.5]. Those idempotents can 
be generated by a choice of two commuting basis elements which square to unity; for the moment we will use $\sigma_{023}$ and $\sigma_{014}$. The set of 4 idempotents is then given by

$$
\begin{array}{ll}
f_{1}=\frac{\left(1+\sigma_{023}\right)\left(1+\sigma_{014}\right)}{4}, & f_{2}=\frac{\left(1+\sigma_{023}\right)\left(1-\sigma_{014}\right)}{4}, \\
f_{3}=\frac{\left(1-\sigma_{023}\right)\left(1-\sigma_{014}\right)}{4}, & f_{4}=\frac{\left(1-\sigma_{023}\right)\left(1+\sigma_{014}\right)}{4} .
\end{array}
$$

Using Eq. (2.9) to make matrix replacements of $\sigma_{023}$ and $\sigma_{014}$ one can find matrix equivalents to these idempotents; those are matrices which have only one non-zero element, located on the diagonal and with unit value.

$S U(3)$ symmetry can now be demonstrated by construction of the 8 generators

$$
\begin{aligned}
& \lambda_{1}=\sigma_{02}\left(f_{1}+f_{2}\right)=\frac{\sigma_{3}+\sigma_{02}}{2}, \\
& \lambda_{2}=\sigma_{03}\left(f_{1}+f_{2}\right)=\frac{-\sigma_{2}+\sigma_{03}}{2}, \\
& \lambda_{3}=f_{1}-f_{2}=\frac{\sigma_{014}-\sigma_{1234}}{2} \\
& \lambda_{4}=-\sigma_{1}\left(f_{2}+f_{3}\right)=\frac{-\sigma_{1}-\sigma_{04}}{2}, \\
& \lambda_{5}=-\sigma_{4}\left(f_{2}+f_{3}\right)=\frac{-\sigma_{4}+\sigma_{01}}{2}, \\
& \lambda_{6}=\sigma_{012}\left(f_{1}+f_{3}\right)=\frac{\sigma_{012}+\sigma_{034}}{2} \\
& \lambda_{7}=-\sigma_{024}\left(f_{1}+f_{3}\right)=\frac{\sigma_{013}-\sigma_{024}}{2} \\
& \lambda_{8}=\frac{f_{1}+f_{2}-2 f_{3}}{\sqrt{3}}=\frac{2 \sigma_{023}+\sigma_{014}+\sigma_{1234}}{2 \sqrt{3}} .
\end{aligned}
$$

These have the following matrix equivalents

$$
\begin{aligned}
& \lambda_{1} \equiv\left(\begin{array}{cccc}
0 & 1 & 0 & 0 \\
1 & 0 & 0 & 0 \\
0 & 0 & 0 & 0 \\
0 & 0 & 0 & 0
\end{array}\right), \quad \lambda_{2} \equiv\left(\begin{array}{cccc}
0 & -\mathrm{j} & 0 & 0 \\
\mathrm{j} & 0 & 0 & 0 \\
0 & 0 & 0 & 0 \\
0 & 0 & 0 & 0
\end{array}\right), \quad \lambda_{3} \equiv\left(\begin{array}{cccc}
1 & 0 & 0 & 0 \\
0 & -1 & 0 & 0 \\
0 & 0 & 0 & 0 \\
0 & 0 & 0 & 0
\end{array}\right) \text {, } \\
& \lambda_{4} \equiv\left(\begin{array}{cccc}
0 & 0 & 0 & 0 \\
0 & 0 & 1 & 0 \\
0 & 1 & 0 & 0 \\
0 & 0 & 0 & 0
\end{array}\right), \quad \lambda_{5} \equiv\left(\begin{array}{cccc}
0 & 0 & 0 & 0 \\
0 & 0 & -\mathrm{j} & 0 \\
0 & \mathrm{j} & 0 & 0 \\
0 & 0 & 0 & 0
\end{array}\right), \quad \lambda_{6} \equiv\left(\begin{array}{cccc}
0 & 0 & 1 & 0 \\
0 & 0 & 0 & 0 \\
1 & 0 & 0 & 0 \\
0 & 0 & 0 & 0
\end{array}\right) \\
& \lambda_{7} \equiv\left(\begin{array}{cccc}
0 & 0 & -\mathrm{j} & 0 \\
0 & 0 & 0 & 0 \\
0 & \mathrm{j} & 0 & 0 \\
0 & 0 & 0 & 0
\end{array}\right), \lambda_{8} \equiv(1 / \sqrt{3})\left(\begin{array}{cccc}
1 & 0 & 0 & 0 \\
0 & 1 & 0 & 0 \\
0 & 0 & -2 & 0 \\
0 & 0 & 0 & 0
\end{array}\right) \text {, }
\end{aligned}
$$


which reproduce Gell-Mann matrices in the upper-left $3 \times 3$ corner 2, 9, 10]. Since the algebra is isomorphic to complex $4 \times 4$ matrix algebra, one expects to find higher order symmetries; Greiner and Müller [9] show how one can add 7 additional generators to those of $S U(3)$ in order to obtain $S U(4)$ and the same procedure can be adopted in geometric algebra. We then define the following additional $S U(4)$ generators

$$
\begin{aligned}
& \lambda_{9}=\sigma_{1}\left(f_{1}+f_{4}\right)=\frac{\sigma_{1}-\sigma_{04}}{2}, \\
& \lambda_{10}=\sigma_{4}\left(f_{1}+f_{4}\right)=\frac{\sigma_{4}+\sigma_{01}}{2}, \\
& \lambda_{11}=-\sigma_{012}\left(f_{2}+f_{4}\right)=\frac{-\sigma_{012}-\sigma_{034}}{2}, \\
& \lambda_{12}=\sigma_{024}\left(f_{2}+f_{4}\right)=\frac{\sigma_{013}+\sigma_{024}}{2}, \\
& \lambda_{13}=\sigma_{3}\left(f_{3}+f_{4}\right)=\frac{\sigma_{3}-\sigma_{02}}{2}, \\
& \lambda_{14}=\sigma_{2}\left(f_{3}+f_{4}\right)=\frac{\sigma_{2}+\sigma_{03}}{2}, \\
& \lambda_{15}=\frac{f_{1}+f_{2}+f_{3}-3 f_{4}}{\sqrt{6}}=\frac{\sigma_{023}-\sigma_{014}-\sigma_{1234}}{\sqrt{6}} .
\end{aligned}
$$

Once again, making the replacements with Eq. (2.9) produces the matrix equivalent generators

$$
\begin{aligned}
\lambda_{9} \equiv\left(\begin{array}{cccc}
0 & 0 & 0 & 1 \\
0 & 0 & 0 & 0 \\
0 & 0 & 0 & 0 \\
1 & 0 & 0 & 0
\end{array}\right), \lambda_{10} \equiv\left(\begin{array}{cccc}
0 & 0 & 0 & -\mathrm{j} \\
0 & 0 & 0 & 0 \\
0 & 0 & 0 & 0 \\
\mathrm{j} & 0 & 0 & 0
\end{array}\right), \lambda_{11} \equiv\left(\begin{array}{cccc}
0 & 0 & 0 & 0 \\
0 & 0 & 0 & 1 \\
0 & 0 & 0 & 0 \\
0 & 1 & 0 & 0
\end{array}\right), \\
\lambda_{12} \equiv\left(\begin{array}{cccc}
0 & 0 & 0 & 0 \\
0 & 0 & 0 & -\mathrm{j} \\
0 & 0 & 0 & 0 \\
0 & \mathrm{j} & 0 & 0
\end{array}\right), \lambda_{13} \equiv\left(\begin{array}{cccc}
0 & 0 & 0 & 0 \\
0 & 0 & 0 & 0 \\
0 & 0 & 0 & 1 \\
0 & 0 & 1 & 0
\end{array}\right), \lambda_{14} \equiv\left(\begin{array}{cccc}
0 & 0 & 0 & 0 \\
0 & 0 & 0 & 0 \\
0 & 0 & 0 & -\mathrm{j} \\
0 & 0 & \mathrm{j} & 0
\end{array}\right) \\
\lambda_{15} \equiv(1 / \sqrt{6})\left(\begin{array}{cccc}
1 & 0 & 0 & 0 \\
0 & 1 & 0 & 0 \\
0 & 0 & 1 & 0 \\
0 & 0 & 0 & -3
\end{array}\right) .
\end{aligned}
$$

The standard model involves the consideration of two independent $S U(3)$ groups, one for colour and the other one for isospin and strangeness; if generators $\lambda_{1}$ to $\lambda_{8}$ apply to one of the $S U(3)$ groups we can produce the generators of the second group by resorting to the basis elements $\sigma_{3}$ 
and $\sigma_{04}$. The new set of 4 idempotents is then given by

$$
\begin{aligned}
& f_{1}=\frac{\left(1+\sigma_{3}\right)\left(1+\sigma_{04}\right)}{4}, \quad f_{2}=\frac{\left(1+\sigma_{3}\right)\left(1-\sigma_{04}\right)}{4}, \\
& f_{3}=\frac{\left(1-\sigma_{3}\right)\left(1-\sigma_{04}\right)}{4}, \quad f_{4}=\frac{\left(1-\sigma_{3}\right)\left(1+\sigma_{04}\right)}{4} .
\end{aligned}
$$

Again a set of $S U(3)$ generators can be constructed following a procedure similar to the previous one

$$
\begin{aligned}
& \alpha_{1}=\sigma_{02}\left(f_{1}+f_{2}\right)=\frac{\sigma_{02}+\sigma_{023}}{2}, \\
& \alpha_{2}=\sigma_{01}\left(f_{1}+f_{2}\right)=\frac{\sigma_{01}+\sigma_{013}}{2}, \\
& \alpha_{3}=f_{1}-f_{2}=\frac{\sigma_{04}-\sigma_{034}}{2}, \\
& \alpha_{4}=\sigma_{2}\left(f_{2}+f_{3}\right)=\frac{\sigma_{2}+\sigma_{024}}{2}, \\
& \alpha_{5}=-\sigma_{1}\left(f_{2}+f_{3}\right)=\frac{-\sigma_{1}-\sigma_{014}}{2}, \\
& \alpha_{6}=\sigma_{4}\left(f_{1}+f_{3}\right)=\frac{\sigma_{4}-\sigma_{03}}{2}, \\
& \alpha_{7}=\sigma_{012}\left(f_{1}+f_{3}\right)=\frac{\sigma_{012}+\sigma_{1234}}{2}, \\
& \alpha_{8}=\frac{f_{1}+f_{2}-2 f_{3}}{\sqrt{3}}=\frac{2 \sigma_{3}+\sigma_{04}+\sigma_{034}}{2 \sqrt{3}} .
\end{aligned}
$$

This new $S U(3)$ group is necessarily independent from the first one because its matrix representation involves matrices with all non-zero rows/columns, while the group generated by $\lambda_{1}$ to $\lambda_{8}$ uses matrices with zero fourth row/column. In the following section we will discuss which of the two groups should be associated with colour.

\section{Monogenic functions}

A monogenic function [4, 7] is defined in any space by the condition $\mathrm{D} \psi=$ 0 . The monogenic condition is more restrictive then the zero Laplacian condition of Eq. 2.21) but obviously a monogenic function always verifies the latter. In particular we can be sure that a monogenic function of $4+1$ space produces a 4-dimensional wave, although the reverse may not be true. Splitting the vector derivative into its time and Euclidean components, we can write the monogenic condition as

$$
\mathrm{D} \psi=\left({ }^{i} \mathrm{D}-\sigma_{0} \partial_{t}\right) \psi=0 .
$$

We expect plane wave solutions and so we try

$$
\psi=\psi_{0} \mathrm{e}^{u\left( \pm p_{0} t+p_{i} x^{i}\right)} ;
$$


here $u$ is a square root of -1 whose characteristics we shall determine, $p_{0}$ is the wave angular frequency and $p_{i}$ are components of a generalized wave vector. When this solution is inserted in the 1st order equation (4.1) we get

$$
\left(\sigma^{i} p_{i} \mp \sigma_{0} p_{0}\right) \psi_{0} u=0 .
$$

The first member can only be zero if $\psi_{0}$ is a multiple of the vector in parenthesis and is nilpotent, i.e.

$$
\sum_{i}\left(p_{i}\right)^{2}-\left(p_{0}\right)^{2}=0
$$

Dirac equation can be recovered from Eq. (4.1) if it is multiplied on the left by $\sigma_{4}$

$$
\left(-\sigma_{40} \partial_{t}+\sigma_{4 m} \partial_{m}+\partial_{4}\right) \psi=0 .
$$

Now note that $\sigma_{40}$ squares to unity and $\sigma_{4 m}$ squares to minus unity, so it is legitimate to make assignments to the Dirac matrices: $\gamma^{0} \equiv-\sigma_{40}, \gamma^{m} \equiv$ $\sigma_{4 m}$. The matrix $\gamma^{5}=\mathrm{i} \gamma^{0} \gamma^{1} \gamma^{2} \gamma^{3}$ becomes, upon substitution, $\gamma^{5} \equiv-\sigma_{4}$.

The last term in the previous equation must be examined with consideration for the proposed solution. Deriving $\psi$ with respect to $x^{4}$ we get

$$
\partial_{4} \psi=p_{4} \psi_{0} u \mathrm{e}^{u\left( \pm p_{0} t+p_{\mu} x^{\mu}\right)} .
$$

Since $u$ will always commute with the exponential, this simplifies to

$$
\partial_{4} \psi=-p_{4} \psi u .
$$

We can then make the further assignments $p_{0}=E$ (energy), $p_{4}=m$ (rest mass), and write

$$
\gamma^{\mu} \partial_{\mu} \psi=-m \psi u \text {. }
$$

We note here that Rowlands 11 has been proposing a nilpotent formulation of Dirac equation for some years, albeit with a different algebra.

The wave function in Eq. (4.2) can now be given a different form, taking in consideration the previous assignments

$$
\psi=A\left(\sigma^{4} m+\mathbf{p} \mp \sigma_{0} E\right) \mathrm{e}^{u( \pm E t+\mathbf{p} \cdot \mathbf{x}+m \tau+\alpha)} ;
$$

where $A$ is the amplitude, $\mathbf{p}=\sigma^{m} p_{m}$ is the 3 -dimensional momentum vector, $\mathbf{x}=\sigma_{m} x^{m}$ is the 3 -dimensional position and $\alpha$ is the phase angle.

Equation (4.1) was written under the assumption of an orthonormed frame but this need not always be the case; the consideration of a refractive index allows the formulation of particle dynamics under gravity.

For gauge fields we modify the vector derivative by introducing a gauge derivative

$$
\mathcal{D}={ }^{\mu} \mathrm{D}+\left(\sigma^{4}+\frac{1}{m} A_{\mu} \sigma^{\mu} u\right) \partial_{4}
$$


Applying the gauge derivative to a general function $\psi$ we get

$$
\mathcal{D} \psi=\left(\frac{1}{m} A_{\mu} \sigma^{\mu} u \partial_{4}+\sigma^{\iota} \partial_{\iota}\right) \psi=0 .
$$

Notice that the $x^{4}$ derivative of $\psi$ includes the $u$ factor which multiplies the same factor present in the gauge derivative operator; when this happens, if additionally $u$ commutes with $\psi$, the parenthesis becomes a vector

$$
g^{\iota} \partial_{\iota} \psi=\left(-A_{\mu} \sigma^{\mu}+\sigma^{\iota} \partial_{\iota}\right) \psi=0 .
$$

Multiplying on the left and replacing with gamma matrices, as above, we get Dirac equation in an electromagnetic field (remember the electron has charge -1$)$.

$$
\gamma^{\mu}\left(\partial_{\mu}-A_{\mu}\right) \psi=-m \psi u .
$$

Obviously the parenthesis in Eq. (4.11) only becomes a vector if the imaginary factor $u$ in the gauge derivative operator is the same as in the wavefunction. When this does not happen, the remaining non-vector term does not play a role in dynamics.

We must now examine in detail what sort of geometrical elements can be used as imaginary $u$ in the solution (4.2); it will be more convenient to write the imaginary as a product of the pseudoscalar and a unitary element, $u=\mathrm{i} h$, since we have already established that each set of 4 idempotents is generated by a pair of commuting unitary basis elements. Let any two such basis elements be denoted as $h_{1}$ and $h_{2}$; then the product $h_{3}=h_{1} h_{2}$ is itself a third commuting basis element. For consistence we choose, as before,

$$
h_{1} \equiv \sigma_{023}, \quad h_{2} \equiv \sigma_{014}
$$

to get

$$
h_{3} \equiv \sigma_{1234},
$$

which commutes with the other two as can be easily verified. The result of this exercise is the existence of triads of commuting unitary basis elements but no tetrads of such elements. We are led to state that a general unitary element is a linear combination of unity and the three elements of one triad

$$
h=a_{0}+a_{1} h_{1}+a_{2} h_{2}+a_{3} h_{3} .
$$

Since $h$ is unitary and the three $h_{m}$ commute we can write

$$
\begin{aligned}
h^{2}= & {\left[\left(a_{0}\right)^{2}+\left(a_{1}\right)^{2}+\left(a_{2}\right)^{2}+\left(a_{3}\right)^{2}\right]+2\left(a_{0} a_{1}-a_{2} a_{3}\right) h_{1} } \\
& +2\left(a_{0} a_{2}-a_{1} a_{3}\right) h_{2}+2\left(a_{0} a_{3}-a_{1} a_{2}\right) h_{3}=1
\end{aligned}
$$

The only form this equation can be verified is if the term in square brackets is unity while all the others are zero. We then get a set of four simultaneous equations with a total of sixteen solutions, as follows: 8 solutions with one 
of the $a_{\mu}$ equal to \pm 1 and all the others zero, 6 solutions with two of the $a_{\mu}$ equal to $-1 / 2$ and the other two equal to $1 / 2$ and 2 solutions with all the $a_{\mu}$ simultaneously $\pm 1 / 2$. The $a_{\mu}$ coefficients play the role of quantum numbers which determine the particular imaginary unit that goes into Eq. (4.2) and consequently into Dirac's equation; these unusual quantum numbers can be combined in order to produce a more conventional set of numbers, as we shall see next. In matrix form these solutions correspond to unitary diagonal matrices with $0,1,2,3$ or 4 negative elements.

There are 16 possible unitary elements defined by Eq. (4.16), corresponding to different solutions of Eq. 4.17). If desired it is possible to express these solutions in terms of the $S U(4)$ generators $\lambda_{3}, \lambda_{8}$ and $\lambda_{15}$ in order to highlight the symmetries. The conversion relations for the generator coefficients are: $\lambda_{3} \rightarrow a_{2}-a_{3}, \lambda_{8} \rightarrow\left(2 a_{1}+a_{2}+a_{3}\right) / \sqrt{3}$ and $\lambda_{15} \rightarrow \sqrt{2 / 3}\left(a_{1}-a_{2}-a_{3}\right)$. In table 1.1 we list all the 16 unitary elements and their respective coefficients.

TABLE 1.1. Coefficients for the various unitary elements.

\begin{tabular}{r|rrr|rrrrr|c}
\hline 1 & $\begin{array}{r}\sigma_{023} \\
\left(a_{1}\right)\end{array}$ & $\begin{array}{r}\sigma_{014} \\
\left(a_{2}\right)\end{array}$ & $\begin{array}{r}\sigma_{1234} \\
\left(a_{3}\right)\end{array}$ & $\lambda_{3}$ & $\lambda_{8}$ & $\lambda_{15}$ & $q$ & $i_{3}$ & Designation \\
\hline 1 & 0 & 0 & 0 & 0 & $0 \sqrt{3}$ & $\sqrt{2 / 3}$ & $0 / 3$ & 0.5 & up \\
0 & 1 & 0 & 0 & 0 & $2 / \sqrt{3}$ & $-\sqrt{2 / 3}$ & $1 / 3$ & 0.5 & anti-down \\
0 & 0 & 1 & 0 & 1 & $1 / \sqrt{3}$ & 1 & 0.5 & positron \\
0 & 0 & 0 & 1 & -1 & $1 / \sqrt{3}$ & $-\sqrt{2 / 3}$ & 0 & -0.5 & anti-up \\
-1 & 0 & 0 & 0 & 0 & 0 & 0 & 0 & 0 \\
0 & -1 & 0 & 0 & 0 & $-2 / \sqrt{3}$ & $-\sqrt{2 / 3}$ & $-2 / 3$ & -0.5 & down \\
0 & 0 & -1 & 0 & -1 & $-1 / \sqrt{3}$ & $\sqrt{2 / 3}$ & $-1 / 3$ & -0.5 & electron \\
0 & 0 & 0 & -1 & 1 & $-1 / \sqrt{3}$ & $\sqrt{2 / 3}$ & -1 & -0.5 & anti-strange \\
$-1 / 2$ & $-1 / 2$ & $1 / 2$ & $1 / 2$ & 0 & 0 & $-\sqrt{3 / 2}$ & $1 / 3$ & 0 & charm \\
$-1 / 2$ & $1 / 2$ & $-1 / 2$ & $1 / 2$ & -1 & $1 / \sqrt{3}$ & $1 / \sqrt{6}$ & $2 / 3$ & 0 & 0 \\
$-1 / 2$ & $1 / 2$ & $1 / 2$ & $-1 / 2$ & 1 & $1 / \sqrt{3}$ & $1 / \sqrt{6}$ & 0 & 0 & \\
$1 / 2$ & $-1 / 2$ & $-1 / 2$ & $1 / 2$ & -1 & $-1 / \sqrt{3}$ & $-1 / \sqrt{6}$ & 0 & 0 & anti-charm \\
$1 / 2$ & $-1 / 2$ & $1 / 2$ & $-1 / 2$ & 1 & $-1 / \sqrt{3}$ & $-1 / \sqrt{6}$ & $-2 / 3$ & 0 & strange \\
$1 / 2$ & $1 / 2$ & $-1 / 2$ & $-1 / 2$ & 0 & 0 & $\sqrt{3 / 2}$ & $-1 / 3$ & 0 \\
$1 / 2$ & $1 / 2$ & $1 / 2$ & $1 / 2$ & 0 & $2 / \sqrt{3}$ & $-1 / \sqrt{6}$ & 1 & 1 & anti-mu \\
$-1 / 2$ & $-1 / 2$ & $-1 / 2$ & $-1 / 2$ & 0 & $-2 / \sqrt{3}$ & $1 / \sqrt{6}$ & -1 & -1 & mu \\
\hline
\end{tabular}

In columns labelled $q$ and $i_{3}$ we make a tentative association between the coefficients $a_{\mu}$, electric charge and isospin through the formulas $q=$ $2 a_{1}+a_{2}+3 a_{3}$ and $i_{3}=\left(a_{0}+a_{1}+a_{2}+a_{3}\right) / 2$. This association is rather speculative and likely to change in the future. Electric charge was assumed to be a count of the number of spatial basis vectors present in the unitary element $h$ and isospin was defined in such a way that it would allow the right combinations for all the particles in two families. Further insight onto the role of the unitary element is needed in order to properly justify the correct association to quantum numbers. The provisional particles' names are included in the last column and we left four positions blank because their significance is unclear for the moment. 


\section{Conclusion}

Using the algebra of 5-dimensional spacetime $G_{4,1}$ as a point of departure, it was possible to derive a number of results of great physical significance from the properties of that algebra. The monogenic condition applied to functions was seen to allow Euclidean space 4-dimensional plane wave solutions but the same condition could be converted into Dirac equation, under certain circumstances, providing an Euclidean interpretation of Dirac spinors. Particle dynamics could be modelled through the definition of a gauge derivative operator, through which the electromagnetic case was successfully formulated.

The exponent in the solutions of the monogenic condition includes a square root of -1 which can be chosen among different possibilities provided by $G_{4,1}$ algebra. An exploration of those possibilities showed that they exhibit the same symmetries as the standard model gauge group, which allowed a tentative mapping to the elementary particles of the two lower families.

\section{REFERENCES}

[1] J. B. Almeida, The null subspace of $G(4,1)$ as source of the main physical theories, in Physical Interpretations of Relativity Theory - IX (London, 2004), physics/0410035

[2] J. B. Almeida, Standard-model symmetry in complexified spacetime algebra, 2003, math.GM/0307165

[3] J. B. Almeida, Euclidean formulation of general relativity, in Number Time and Relativity (Moscow, 2004), physics/0406026

[4] C. Doran and A. Lasenby, Geometric Algebra for Physicists (Cambridge University Press, Cambridge, U.K., 2003).

[5] D. Hestenes and G. Sobczyk, Clifford Algebras to Geometric Calculus. A Unified Language for Mathematics and Physics, Fundamental Theories of Physics (Reidel, Dordrecht, 1989).

[6] S. Gull, A. Lasenby, and C. Doran, Imaginary numbers are not real. The geometric algebra of spacetime, Found. Phys. 23, 1175, 1993, URL http://www.mrao.cam.ac.uk/ clifford/publications/abstracts/imag_numbs.html

[7] A. Lasenby and C. Doran, Physical applications of geometric algebra, handout collection from a Cambridge University lecture course, 1999, URL http://www.mrao.cam.ac.uk/ clifford/ptIIIcourse/course99/

[8] P. Lounesto, Clifford Algebras and Spinors, vol. 286 of London Mathematical Society Lecture Note Series (Cambridge University Press, Cambridge, U.K., 2001), 2nd ed.

[9] W. Greiner and B. Müller, Quantum Mechanics: Symmetries (Springer, Berlin, 2001), 2nd ed.

[10] W. N. Cottingham and D. A. Greenwood, An Introduction to the Standard Model of Particle Physics (Cambrige University Press, Cambridge, U.K., 1998). 
[11] P. Rowlands, The nilpotent Dirac equation and its applications in particle physics, 2003, quant-ph/0301071

José B. Almeida

Universidade do Minho

Physics Department

4710-057 Braga

Portugal

E-mail: bda@fisica.uminho.pt

Submitted: TBA; Revised: October 23, 2018. 